\title{
2016 Reviewers
}

\author{
Abonyi, János \\ Ádám, Norbert \\ Alexik, Mikulas \\ Ancza, Erzsébet \\ Ando, Matyas \\ Anh, Anh Le \\ Antal, Margit \\ Babic, Frantisek \\ Balas, Valentina Emilia \\ Bartak, Roman \\ Batyrshin, Ildar \\ Benczúr, András \\ Beneda, Károly \\ Benesova, Wanda \\ Berenguel, Manuel \\ Biro, Miklos \\ Bitó, János \\ Bobak, Martin \\ Borsa, Judit \\ Brown, John N. A. \\ Bucko, Jozef \\ Bukovics, István \\ Bundzel, Marek \\ Butka, Peter \\ Búza, Antal \\ Cádrik, Tomáš \\ Csabai, István \\ Csákány, Rita \\ Csernátonyi, Zoltán \\ Czifra, Árpád \\ Do, Tien V. \\ Dobos, László \\ Dobránszky, János \\ Dömötör, Ferenc \\ Dragan, Antic \\ Drexler, Dániel András
}

Drotár, Peter

Duchon, Frantisek

Eigner, György

Ekler, Péter

Elmenreich, Wilfried

Ferenci, Tamás

Firstner, István

Fogarassy-Vathy, Ágnes

Fridli, Sándor

Fullér, Róbert

Gazda, Juraj

Giang, Nguyen

Haraksim, Rudolf

Holik, Ildikó

Horváth, Csaba

Horváth, Richárd

Hreno, Jan

Hudec, Ladislav

Jadlovská, Slávka

Jeszenszky, Peter

Józsa, Lajos

Jung, Jason

Kádár, István

Kajtár, László

Kaptay, George

Karlovitz, Tibor János

Kasanicky, Tomas

Kiss, Rita

Klešč, Marián

Koncsik, Zsuzsanna

Kósi, Krisztián

Kovács, Attila

Kovács, László

Kovács, Levente

Kovács, Szilveszter

Kovács-Coskun, Tünde 
Kovarova, Alena

Krajci, Stanislav

Krómer, István

Kucera, Markus

Kuzsella, László

Laki, Sándor

Lazányi, Kornélia

Liščinský, Pavol

Luca, Mihaela

Machado, José

Markopoulos, Angelos

Márkus, Ferenc

Maros-Berkes, Mária

Marosi, lldikó

Mátray, Péter

Mertinger, Valéria

Michelberger, Pál

Micsik, Andras

Milosavljevic, Cedomir

Molnár, Bálint

Morva, György

Nagy, Dénes Ákos

Nagy, István

Ocelíková, Eva

Palik, Mátyás

Palúch, Stanislav

Paralic, Jan

Piazolo, Marc

Pintér, István

Plavka, Ján

Pleva, Matus

Pokorádi, László

Porkoláb, Zoltán

Precup, Radu-Emil

Puheim, Michal

Rácz, Ervin

Radac, Mircea-Bogdan

Reicher, Regina

Restas, Agoston
Réveszová, Libuša Sali, Attila

Sallai, Gyula

Sánta, Imre

Sarnovsky, Martin

Semanisin, Gabriel

Sergyán, Szabolcs

Sidló, Csaba István

Simonak, Slavomir

Simsik, Dusan

Škrinárová, Jarmila

Sobota, Branislav

Son, Hua Nam

Szabó, Imre

Szabó, József Zoltán

Szabó, László Zsolt

Szabó, Tamás

Szénási, Sándor

Szlivka, Ferenc

Szücs, Gábor

Takács-György, Katalin

Tar, József

Tejfel, Máté

Tomoriova, Beata

Tóth, Péter

Tóth-Laufer, Edit

Trinh, Anh Tuan

Trohák, Attila

Uj, József

Vajk, István

Vámossy, Zoltán

Varga, Béla

Varga, Péter János

Varga, Viorica

Vas, László

Vassileva, Bistra

Vilanova, Ramon

Windisch, Gergely

Zdešar, Andrej 